\title{
The UPR in atherosclerosis
}

\author{
Alex X. Zhou and Ira Tabas \\ Department of Medicine, Columbia University, New York, NY, USA
}

\section{Abstract}

Multiple systemic factors and local stressors in the arterial wall can disturb the functions of endoplasmic reticulum (ER), causing ER stress in endothelial cells (ECs), smooth muscle cells (SMCs), and macrophages during the initiation and progression of atherosclerosis. As a protective response to restore ER homeostasis, the unfolded protein response (UPR) is initiated by three major ER sensors: protein kinase RNA-like ER kinase (PERK), inositol-requiring protein 1a (IRE1a), and activating transcription factor 6 (ATF6). The activation of the various UPR signaling pathways displays a temporal pattern of activation at different stages of the disease. The ATF6 and IRE1 a pathways that promote the expression of protein chaperones in ER are activated in ECs in athero-susceptible regions of prelesional arteries and before the appearance of foam cells. The PERK pathway that reduces ER protein client load by blocking protein translation is activated in SMCs and macrophages in early lesions. The activation of these UPR signaling pathways aims to cope with the ER stress and plays a pro-survival role in the early stage of atherosclerosis. However, with the progression of atherosclerosis, the extended duration and increased intensity of ER stress in lesions lead to prolonged and enhanced UPR signaling. Under this circumstance, the PERK pathway induces expression of death effectors, and possibly IRE1a activates apoptosis signaling pathways, leading to apoptosis of macrophages and SMCs in advanced lesions. Importantly, UPR-mediated cell death is associated with plaque instability and the clinical progression of atherosclerosis. Moreover, UPR signaling is linked to inflammation and possibly to macrophage differentiation in lesions. Therapeutic approaches targeting the UPR may have promise in the prevention and/or regression of atherosclerosis. However, more progress is needed to fully understand all of the roles of the UPR in atherosclerosis and to harness this information for therapeutic advances.

\section{Keywords}

Atherosclerosis; Unfolded protein response; Endoplasmic reticulum; Stress

\section{Introduction}

\begin{abstract}
Atherosclerosis is the primary pathological process that leads to cardiovascular disease, the leading cause of mortality and illness in developed countries [1]. Intensive studies on the cellular and molecular mechanisms of atherosclerosis over the past decades have led to a consensus view that retention of lipoproteins and a subsequent maladaptive inflammatory response are major contributors to atherogenesis. Although therapeutic interventions targeting lipoprotein metabolism have made great progress, atherosclerosis remains a common and challenging illness. Many aspects of atherogenesis still remain incompletely understood, and novel therapeutic targets to prevent the disease or cause its regression need to be identified and validated [2].
\end{abstract}


Work over the last decade has revealed that endoplasmic reticulum (ER) stress is an important event during the initiation, progression, and clinical progression of atherosclerosis [3]. The ER is the cellular organelle responsible for the proper folding and maturation of secretory and membrane proteins. In addition, the ER is also the major intracellular reservoir of calcium, and its membrane is the place where many rate-limiting enzymes of lipid biosynthesis are located. Stimuli such as oxidative stress, ischemic insult, disturbances in calcium homeostasis, and enhanced expression of normal and/or folding-defective proteins lead to the accumulation of unfolded proteins and other perturbations in ER function [4]. As an adaptive action, the unfolded protein response (UPR) is triggered to maintain ER homeostasis. In mammalian cells, the UPR is triggered by the activation of at least three major stress sensors located on the ER membrane: protein kinase RNA-like ER kinase (PERK), inositol-requiring protein 1a (IRE1a), and activating transcription factor 6 (ATF6) [5]. These sensors are maintained in an inactive state under non-stress conditions through an association with the protein chaperone glucose-regulated protein 78 (GRP78)/BiP in the ER lumen. However, upon aggregation of unfolded proteins and other ER stress conditions, GRP78 dissociates from these sensors, allowing their activation and initiation of UPR signaling.

One critical action of UPR signaling is to reduce protein synthesis as a means to re-establish homeostasis. In particular, activated PERK blocks general protein synthesis by phosphorylating (p) eukaryotic initiation factor $2 a($ IF $2 a)$, and IRE1a may cause degradation of certain mRNAs through a process known as regulated IRE1-dependent decay (RIDD). Second, a coordinately regulated transcriptional network is activated, involving three distinct UPR transcriptional factors, ATF4, ATF6, and X-box binding protein 1 (XBP1), to regulate the expression of genes required to restore ER homeostasis. Ironically, ATF4 escapes translational inhibition through a mechanism involving alternative openreading frame usage in the $5^{\prime}$ UTR of the Atf4 transcript. ATF4 translocates to the nucleus and activates genes that encode amino acid transporters and genes that protect against oxidative stress. ATF6 is activated by limited proteolysis after its translocation from the ER to the Golgi apparatus. Active ATF6 regulates the expression of ER chaperones and XBP1. XBP1 must undergo mRNA splicing to achieve its active form, which is carried out by IRE1a. Spliced XBP1 protein (sXBP1) translocates to the nucleus and controls the transcription of ER chaperones and components of the ER-associated degradation (ERAD) process. ERAD functions at the post-translational level by targeting misfolded proteins of ER for ubiquitination and subsequent degradation by the proteasome. Together, the three UPR signaling branches coordinately act at the transcriptional, post-transcriptional, and post-translational level to resolve ER stress by blocking general protein synthesis, enhancing the folding capacity, and initiating degradation of potentially cytotoxic protein aggregates [5-7].

The development of atherosclerosis is a chronic, complex process involving a number of systemic and arterial-wall risk factors. Atherogenesis begins with the retention of apolipoprotein B-containing lipoproteins in focal areas of the arterial subendothelium. The retained and subsequently modified subendothelial lipoproteins, as well as other risk factors like shear stress, contribute to the activation of vascular endothelial cells (ECs). Activated endothelium expresses adhesion molecules that cause monocytes to stick to the endothelium and infiltrate into the vascular wall, where they differentiate into macrophages and ingest retained lipids to become "foam cells." Macrophages, together with the T cells, mast cells, and other inflammatory cells that enter developing atherosclerotic lesions, contribute to a maladaptive inflammatory response that drives atherosclerosis $[8,9]$. The cytokines and growth factors produced by these immune cells promote a change in the localization and phenotype of smooth muscle cells (SMCs). In response to atherogenic stimuli, SMCs undergo a conversion from a "contractile" phenotype to a "synthetic" phenotype. The 
synthetic SMCs produce a massive amount of extracellular matrix in the lesions. SMCs and extracellular matrix contribute to the further retention of lipoproteins and immune cells during the progression of atherosclerosis, while in advanced lesions they appear to help stabilize plaques through the synthesis of a fibrous cap overlying the lesions [10]. Eventually, the increasingly complex lesion consists of immune cells, vascular ECs and SMCs, connective-tissue elements, lipids, and debris. In the center of an atheroma, foam cells and extracellular lipid droplets form a core region, which is surrounded by a cap of SMCs and a collagen-rich matrix. T cells, macrophages, and mast cells continue to infiltrate the progressive lesion and are particularly abundant in the shoulder region where the atheroma grows [11]. Importantly, only a small percentage of lesions lead to acute clinical manifestations resulting from plaque rupture and subsequent thrombosis and vessel occlusion [12]. A common feature of these unstable plaques is the accumulation of apoptotic immune cells and vascular cells [13, 14]. Normally apoptotic cells are removed by phagocytic cells ("efferocytosis"). As lesions progress, these apoptotic cells become "secondarily necrotic" due to defective efferocytosis. The cumulative effect of these late lesional events is the generation of a necrotic core, which, in concert with pro-atherogenic effects of residual surviving macrophages, promotes further inflammation, plaque instability, and thrombosis [13].

Although the UPR is initiated as an adaptive response, an unresolved UPR response can lead to apoptotic cell death, perhaps designed to get rid of potentially damaging cells through efferocytosis. In addition, components of the UPR regulate various physiological and pathological processes, ranging from lipid and cholesterol metabolism and energy homeostasis, to inflammation and cell differentiation [15]. Inflammation and apoptosis are key pathological processes in atherosclerosis. The potential role of UPR in regulating these processes, together with evidence showing activation of UPR in early and advanced atherosclerotic lesions, suggest a link of UPR to the progression and clinical complications of atherosclerosis. This review is designed to provide an overview of the evidence for UPR activation in atherosclerosis, the possible roles the UPR may play in atherosclerosis, and the therapeutic potentials to treat atherosclerosis by targeting the UPR.

\section{Evidence of UPR activation in atherosclerosis (Fig. 1)}

Advanced atherosclerotic plaques provide a pathophysiological environment that can cause ER stress and activate the UPR, which is due, at least in part, to the presence of oxidized lipids, inflammation, and metabolic stress [11]. ER stress and UPR activation markers have been observed in both human and animal atherosclerotic lesions. Myoishi et al. [16] examined the histological sections of human atherosclerotic coronary artery lesions obtained at autopsy or after directional coronary atherectomy (DCA). The lesions in the human coronary specimens from autopsy were classified by histochemistry as being diffuse intimal thickening, fibrous plaques, thick-cap atheroma, thin-cap atheroma, or ruptured plaques. The latter two classifications are associated with acute clinical syndromes, while the former three types of plaques are not. Both SMCs and macrophages exhibited a markedly increased expression of the ER chaperones GRP78 and GRP94 and CCAAT/enhancer binding protein homologous protein (CHOP) in thin-cap atheroma and ruptured plaques compared with intimal thickening, fibrous plaques, and thick-cap atheroma. The DCA specimens in the study came from patients with stable angina pectoris (SAP) or unstable angina pectoris (UAP) according to clinical manifestation. Again, morphometric analysis demonstrated that the numbers of KDEL- and CHOP-positive cells were significantly higher in patients with UAP than SAP. Similarly, the mRNA expression of GRP78 and CHOP were higher in UAP patients. Although this study cannot make any conclusions about causation, it provides evidence that activation of UPR is associated with the severity and clinical complications of atherosclerosis in humans. 
Causation studies linking the UPR to atherosclerosis have relied upon genetically engineered mouse models of atherosclerosis. Mouse models of atherosclerosis faithfully recapitulate early lesion development, and although plaque rupture does not occur in these models, they do develop features that are known to be associated with plaque rupture in humans, including necrotic cores and intimal cell apoptosis. The activation of UPR signaling is found at different stages of atherosclerosis in mice. Zhou et al. assessed the expression of UPR markers in three stages of lesional macrophages at the aortic root in Apoe ${ }^{-/-}$mice: intimal macrophages, macrophage foam cells within the fatty streak, and macrophage foam cells in complex lesions [17]. Protein disulphide isomerase and the ER chaperones GRP78 and calreticulin were found to be highly expressed in both non-lipid-loaded macrophages and macrophage foam cells, as well as in the medial SMCs. Phospho-PERK was observed at all stages of lesional macrophage development. In advanced lesions, cells within the cap were strongly positive for phospho-PERK, and intimal SMCs were occasionally positive for phospho-PERK. CHOP immunostaining of murine atherosclerotic lesions can yield a falsepositive signal, but Western blot analysis of lesional extracts demonstrate strong CHOP expression in advanced lesions, consistent with the aforementioned study with human plaques. Consistent with these data, laser-captured RNA from macrophage-rich regions of advanced murine plaques show high levels of Chop mRNA [18]. T cell death-associated gene 51 (TDAG51), which is an ER stress-inducible protein that promotes detachmentmediated apoptotic cell death [19], was observed at all stages of lesions. Finally, both spliced (active) and unspliced (latent) forms of the XBP1 protein were increased in the advanced lesion group.

\section{Lipid metabolism and UPR}

Macrophages are capable of ingesting apolipoprotein-B (apoB)-containing lipoproteins and transporting ingested lipoproteins from late endosomes to the ER, where cholesterol is esterified to form inert lipid droplets. However, macrophages in advanced lesions appear to have reduced capability of esterifying cholesterol, leading to intracellular accumulation of unesterified (free) cholesterol [20]. In this setting, trafficking of free cholesterol to the normally cholesterol-poor ER membrane alters the physicochemical properties of this membrane and leads to disruption of ER function and activation of the UPR [21]. A cytosolic lipid chaperone called macrophage fatty acid-binding protein-4 (aP2) regulates cellular lipid trafficking and metabolism. Human THP-1 monocytes cultured with oxidized low-density lipoprotein (oxLDL) showed induction of aP2 expression [22], and aP2deficient macrophages showed significantly reduced p-PERK, P-eIF2a, and XBP1 splicing upon treatment with palmitate [23]. Thus, aP2 appears to be a mediator between lipotoxic signals and macrophage ER stress. The role of aP2 in this process involves suppression of activation of liver X receptor (LXR), which up-regulates key lipogenic enzymes that convert saturated fatty acids into less toxic monounsaturated fatty acids [23]. In $A p o e^{-/-}$mice, total or macrophage-specific aP2-deficiency provides significant protection against atherosclerosis [24].

\section{Shear stress induced UPR}

Endothelial function and dysfunction are central to the focal origin and regional development of atherosclerosis. Atherosclerotic lesions generally occur at arterial bends, characterized by flow separation and low shear stress. Using a nonatherosclerotic pig model, Davies' group compared the transcript profiles of endothelial cell samples isolated from discrete coronary and non-coronary arterial regions of varying susceptibilities to atherosclerosis [25]. The analysis revealed markers of ER stress activation in atherosusceptible vs. athero-resistant regions. The differentially expressed ER-associated genes were functionally categorized into mRNA processing, protein folding, protein transport and quality control, protein degradation, and ER lipid biosynthesis [26]. In athero-susceptible 
regions, IRE1a, ATF6, and XBP1, but not PERK or eIF2a, were activated in concert with a higher transcript expression of protein folding enzymes and chaperones. Moreover, expression levels of ATF4 and CHOP mRNA, but not protein, were increased. These data suggest UPR activation in endothelial cells occurs at the very early stage of atherosclerosis. At this stage, UPR may be functioning as a protective response to the disturbed blood flow and low shear stress. However, if UPR activation is prolonged or exacerbated by other ER stress inducers, such as hypercholesterolemia, it may be detrimental to the survival of the endothelium. For example, overexpression of spliced XBP1 induced endothelial loss from blood vessels during ex vivo culture of mouse artery segments. Importantly, adenoviral gene transfer of spliced XBP1 to the vessel wall of $A p o e^{-/-}$mice resulted in development of atherosclerotic lesions after aorta isografting [27].

\section{Hyperhomocysteinemia-induced UPR}

Epidemiologic studies suggest that moderately elevated total serum homocysteine (tHcy) levels are prevalent in the general population and, though still controversial [28], may be associated with an increased risk for cardiovascular disease, independent of classic cardiovascular risk factors [29]. Studies using genetic- or diet-induced animal models of hyperhomocysteinemia (HHcy) have demonstrated a causal relationship between HHcy and accelerated atherosclerosis [30, 31]. HHcy can be induced in animal models by using diets supplemented with methionine, which is metabolized to homocysteine after being coingested with cholesterol. In these models, HHcy increases atherosclerotic lesion size, and was found to enhance the expression of ER stress markers, including GRP78/94 and phospho-PERK, in the advanced lesions of $A p o e^{-/-}$mice. Interestingly, heat shock protein 47 (HSP47), an ER-resident molecular chaperone involved in collagen folding and secretion, was also increased in advanced lesions of HHcy mice. In advanced lesions, GRP78/94 and HSP47 were predominantly localized to the smooth muscle cell-rich fibrous cap, suggesting activation of the UPR in SMCs [31]. SMCs are the major producers of extracellular matrix proteins in atherosclerotic lesions, and this high level of protein synthesis may increase the protein client load in ER and thereby cause UPR activation. Another mouse study suggested that homocysteine may specifically target the PERK branch of the UPR in macrophages. Interestingly, the expression of GRP78/94 was comparable between mice fed with or without methionine supplementation despite of methionine-induced HHcy [32]. The finding that HHcy induces an UPR was also demonstrated in a rabbit model, in which endothelial CHOP expression was induced in association with EC apoptosis and larger plaque size [33]. Addition of taurine in the diet, which blocks absorption of methionine, did not improve the lipid profile but markedly inhibited the increase in plasma methionine and Hcy and was associated with a decrease in endothelial CHOP expression [33].

\section{UPR and apoptosis in atherosclerotic lesions}

Apoptosis is a prominent event in advanced atheroma and a major cause of plaque rupture. Sustained UPR activation has been linked to apoptosis in multiple cell types including pancreatic cells, liver cells, immune cells, and vascular cells [34]. UPR activation in advanced atherosclerosis is associated with intimal cell apoptosis in both human and animal studies. In the aforementioned human study, thin-cap atheroma and ruptured plaques, or lesions from patients with unstable angina pectoris, displayed significantly higher levels of TUNEL-positive (apoptotic) macrophages and SMCs compared with earlier or more stable lesions [16]. Correspondingly, these unstable plaques also showed a higher expression of CHOP, and a large portion of the CHOP-positive cells were TUNEL positive. Similar findings occur in the advanced lesions of fat-fed $\mathrm{Apoe}^{-/-}$mice [17]. The oxysterol 7ketocholesterol was present at higher levels in human thin-cap atheroma compared with thick-cap atheroma. 7-Ketocholesterol, which is the most abundant oxysterol in human 
atheroma, can cause oxidative and ER stress. Treatment of cultured coronary artery SMCs or THP-1 monocytes with 7-ketocholesterol induced the upregulation of ER chaperones and apoptosis [16].

Even in advanced lesions, only a small percentage of cells are apoptotic at any one point in time. One study found that early lesional macrophages accumulated FC but that these cells were not apoptotic [17]. One possible explanation is that apoptotic cells are rapidly cleared by neighboring macrophages via efferocytosis [13]. On the other hand, this may reflect the pro-survival function of early UPR activation. Some UPR markers found in early lesions including GRP78, GRP94, and spliced XBP1 are activated after human blood monocytes are differentiated into macrophages by macrophage-colony stimulating factor (M-CSF) in vitro, and this process actually protects macrophages from ER stress-induced apoptosis [35]. The conversion of UPR signaling from pro-survival to pro-apoptotic is probably a slowly evolving process in vivo as manifested by the delayed expression of CHOP. Moreover, the initial ER stress response is often accompanied by other pro-survival pathways in early lesions, such as interferon- $\beta$, Akt (or protein kinase B), NF- $\kappa \mathrm{B}$, p38a, extracellular signalregulated kinase (ERK), and autophagy [3]. Apoptosis eventually occurs when the balance between pro-survival and proapoptotic functions breaks down.

The causative role of CHOP in macrophage apoptosis in advanced atherosclerosis has been established by studying athero-prone mice with genetically engineered deletion of CHOP. In particular, when CHOP knockout mice were bred with $\mathrm{Ldlr}^{-/-}$or $A p o e^{-/-}$mice and fed with Western diet, CHOP deficiency was associated with somewhat less total lesion area and much less necrotic area [18]. Macrophage apoptosis in advanced lesions of CHOP knockout mice was significantly reduced $[18,36]$. The peritoneal macrophages isolated from CHOP knockout mice also displayed less apoptosis compared to WT cells when stressed with 7ketocholesterol or oxLDL plus the peroxynitrite donor SIN-1. As mice generally do not develop spontaneous plaque rupture in aorta, an artificial plaque rupture model was generated by the ligation and cuff placement method [36]. The number of plaque disruption events was markedly prevented by CHOP knockout in this model, supporting the hypothesis that activation of $\mathrm{CHOP}$ contributes to the instability of atherosclerotic plaques. Moreover, bone marrow-derived cells seem to be the major player in CHOP-dependent plaque disruption, as transplantation of CHOP knockout donor bone marrow into $\mathrm{Chop}^{+/+}$mice was sufficient to reduce plaque disruption. Interestingly, another bone marrow transplantation study reported that recipient CHOP deficiency significantly suppressed both cuff injuryinduced neointimal formation and hypercholesterolemia-induced atherosclerotic plaque formation to a greater extent than donor CHOP deficiency, although macrophage apoptosis was not studied [37]. This suggests that the role of CHOP in atherosclerosis may be not limited to macrophage apoptosis and that SMC and/or EC CHOP may function in atherogenic processes.

\section{Mechanisms of UPR-mediated apoptosis relevant to atherosclerosis ER stress-mediated apoptosis in macrophages}

ER stress-induced apoptosis in macrophages has been extensively studied by a number of laboratories and, as described above, is relevant to advanced atherosclerosis. ER stress in cultured macrophages can be induced by forcing intracellular accumulation of unesterified cholesterol or by exposure to "athero-relevant" ER stress inducers like oxysterols [20, 38, 39]. A comprehensive understanding of the pro-apoptotic actions of prolonged $\mathrm{CHOP}$ expression has been obtained based on both in vitro studies and aforementioned genetically modified animal studies (reviewed in [34]). One prominent mechanism involves the induction of ER oxidase 1a (ERO1a) by CHOP in ER-stressed macrophages, which activates inositol triphosphate receptor (IP3R)-mediated release of calcium into the cytosol. 
This increase in cytosolic calcium activates a kinase called calcium/calmodulin-dependent protein kinase II (CaMKII), which triggers apoptosis execution pathways through a number of mechanisms. These mechanisms include induction of the Fas death receptor, mitochondrial release of apoptogens, a pro-apoptotic pathway involving signal transducer and activator of transcription-1 (STAT1), and NADPH oxidase-mediated ROS generation [40-42].

In addition to this well-characterized mechanism based on macrophage studies, two other molecular mechanisms of CHOP-induced cell apoptosis have also been identified. First, CHOP regulates the expression of anti-apoptotic and proapoptotic members of BCL family. CHOP down-regulates the anti-apoptotic BCL family member protein Bcl-2 and Bcl-2 transduction of these cells rescues the cells from both oxidative stress and apoptosis [43-45]. On the other hand, CHOP up-regulates the pro-apoptotic protein Bim and PUMA, leading to mitochondria-dependent apoptosis [46, 47]. Second, CHOP induces the expression of its transcriptional target DNA damage-inducible protein 34 (GADD34), which promotes the dephosphorylation of eIF2 $\alpha$ and thus restores protein translation. The increased protein client load in cells with high levels of protein synthesis can further perturb ER function leading to cell death [48]. Whether any of these mechanisms is relevant to cell death in atherosclerosis remains to be determined.

In most of in vitro experiments, a high dose of ER stress inducers is used, which can probably trigger the full spectrum of UPR signaling. However, macrophages in atherosclerotic lesions are likely exposed to a more subtle ER stress environment and may require a "second hit" to become apoptotic. In vitro and in vivo data suggest that pattern recognition receptors (PRRs), notably scavenger receptors and toll-like receptors (TLRs), can function as second hits to trigger apoptosis in macrophages exposed to low levels of ER stress [3]. For example, low doses of 7-ketocholesterol, thapsigargin, or the peroxynitrite donor SIN-1 showed synergetic effects on macrophage apoptosis when combined with PRR activators that are known to be present in advanced lesions, such as oxidized phospholipids, oxidized LDL, and saturated fatty acids [42]. The mechanism by which PRR activation tips the balance toward apoptosis in ER-stressed macrophages involves amplification of cell death pathways, such as NADPH oxidase-mediated ROS generation, and suppression of ER stress-induced compensatory cell survival pathways [42, 49, 50].

\section{ER stress-mediated apoptosis in ECs and SMCs}

In cultured ECs, homocysteine-induced CHOP induction and apoptosis can be prevented by a dominant negative form of IRE1 [51]. IRE1 causes XBP-1 splicing and activation, and the overexpression of spliced XBP1 induces apoptosis in cultured human umbilical vein endothelial cells (HUVEC) and endothelial loss from blood vessels ex vivo [27]. Interestingly, this effect was found to be mediated by the down-regulation of the adherens junction molecule VEcadherin. Reconstitution of VE-cadherin significantly increased the survival of HUVECs in these cells. Homocysteine-induced EC death can also be mediated by the induction of TDAG51, which is up-regulated by phosphorylated eIF2a [19]. Overexpression of TDAG51 in ECs decreases cell adhesion and promotes detachmentmediated apoptosis. Furthermore, glycated and oxidized LDL, two common ER stressors present in atherosclerotic lesions, induce prolonged ER stress in cultured ECs by inducing oxidative stress and inhibiting the ER calcium pump SERCA [52].

In cultured SMCs, increased CHOP expression and apoptosis have been observed following exposure to 7-ketocholesterol [53] and unesterified cholesterol [54]. Silencing CHOP by small interfering RNA decreased 7-ketocholesterol-induced cell death [16]. 7-

Ketocholesterol also activated the IRE-1/c-Jun-N-terminal kinase (JNK) /AP1 signaling pathway, which led to increased expression of Nox-4 and subsequent oxidative stress. 
Silencing Nox-4 expression significantly reduced the 7-ketocholesterol-induced production of ROS and abolished apoptotic events [53].

As mentioned above, studies in ECs and SMCs have suggested a role for IRE1a in apoptosis. Several molecular mechanisms of activated IRE1a-induced apoptosis have been explored and need to be examined in athero-relevant studies. First, IRE1a's RNase can cause endonucleolytic decay of many ER-localized mRNAs, including those encoding chaperones. Thus persistent IRE1a activation can signal an inability to adapt to ER stress and trigger a switch to apoptosis [55]. Second, activation of IRE1a can recruit the adaptor protein TNFR-associated factor 2 (TRAF2), which results in the activation of the apoptosis signal-regulating kinase 1 (ASK1) pathway and its downstream target JNK [56]. JNK can induce apoptosis through a BAX/BAK-dependent mitochondrial pathway. For instance, JNK disrupts the balance between the pro- and anti-apoptotic factors upstream of BAX/BAK via activation of the pro-apoptotic proteins BIM and BMF [57] and deactivation of the antiapoptotic protein BCL-2 [58]. Third, sustained IRE1a RNase activation induces caspase-2 (CASP2) protein via a rapid decay of select microRNAs that normally repress translation of Casp2 mRNA [59]. Once activated, CASP2 cleaves the pro-apoptotic BCL-2 protein BID, which then localizes to mitochondria to induce BAX/BAK-dependent apoptosis [60]. Fourth, the decay of miR-17 by hyperactivated IRE1a also causes the elevation of thioredoxin-interacting protein (TXNIP) expression. Elevated TXNIP activates the NLRP3 inflammasome, which cleaves procaspase- 1 to its active form, in turn causing maturation and secretion of IL-1 $\beta$, thus promoting sterile inflammation and apoptosis [61].

\section{UPR and inflammation in atherosclerosis}

Growing evidence suggests that signaling pathways in the UPR and inflammation are interconnected through various mechanisms. First, PERK-mediated translational attenuation leads to shortage of I $\kappa \mathrm{B}$ due to its shorter half-life, thereby freeing NF- $\kappa \mathrm{B}$ to translocate to the nucleus. Second, the IRE1a-TRAF2 complex recruits and activates JNK and IkB kinase (IKK). Activated JNK phosphorylates the transcription factor AP1. Activated IKK phosphorylates I $\kappa B$ leading to its degradation and activation of NF- $\kappa$ B. Activated AP1 and $\mathrm{NF}-\mathrm{\kappa B}$ induce the transcription of a large number of inflammatory mediators. Third, an increase in the protein folding load in the ER can lead to the accumulation of ROS, which may initiate an inflammatory response. (reviewed in [62]) Importantly, the links between UPR and inflammation are evident in various inflammation-driven metabolic diseases [63].

Inflammation and UPR are particularly important in atherosclerosis. For example, accumulation of free cholesterol (FC) in macrophages leads to the induction and secretion of tumor necrosis factor-a (TNFa) and interleukin-6 (IL-6), which is mediated by FC-induced activation of the IKK/NF- $\kappa$ B pathway as well as activation of MKK3/p38, Erk1/2, and JNK1/2 mitogen-activated protein kinases (MAPK). The activation of all of the signaling pathways and induction of both cytokines require cholesterol trafficking to ER. The CHOP branch of the UPR signaling is required for Erk1/2 activation and IL- 6 induction. In contrast, one or more other ER-related pathways are responsible for activation of $\mathrm{p} 38$, $\mathrm{JNK} 1 / 2$, and IKK/NF- $\mathrm{KB}$ and for the induction of TNFa [64]. Interestingly, in contrast to the aforementioned IRE1a-mediated activation of the nucleotide oligomerization domain receptor protein 3 (NLRP3) inflammasome and the subsequent release of the proinflammatory cytokine IL-1 $\beta$ in THP-1 monocytes [61], another study found that the activation of NLRP3 inflammasome caused by the ER stressors brefeldin A (BFA), tunicamycin, and thapsigargin in THP-1 monocytes and human macrophages was independent of PERK, IRE1a, or ATF6 [65]. Further studies are required to characterize this unconventional ER stress response. 
Evidence gained from ECs also supports a link between ER stress and inflammation. Studies using human aortic ECs found that UPR-related genes were induced by the oxidized phospholipid oxidized 1-palmitoyl-2-arachidonoyl-sn-glycero-3-phosphocholine (oxPAPC)treated human aortic ECs [66, 67]. The ATF4 and XBP1 branches of the UPR were essential mediators of basal and oxPAPC-induced expression of the inflammatory cytokines IL-8, IL-6, MCP1, and the chemokine CXC motif ligand 3 (CXCL3) [67, 68]. In vivo, ATF3 and ATF4 were strongly expressed in ECs in the more inflammatory shoulder area of human coronary atherosclerotic lesions rather than in the fibrous cap area. The shoulder region also showed a high level of the oxidized phospholipid oxPAPC, suggesting a link between oxPAPC and the UPR [68].

\section{ER stress and macrophage differentiation}

Macrophage phenotypic variation is evident in atherosclerosis as it is in other inflammatory conditions [69]. Although this variation is often denoted by the letter " $\mathrm{M}$ " followed by a number, such as"M1" for so-called classically activated macrophages and "M2" for socalled alternatively activated macrophages, a clear delineation is usually not observed [70]. Nonetheless, this notation appears in the literature and will be used below. In chow-fed Apoe $^{-/-}$mice, lesioninfiltrated macrophages of young mice exhibit predominantly the M2 phenotype, whereas M1 macrophages are dominant in more advanced lesions of aged mice [71]. ER stress was recently found to be a key regulator of macrophage differentiation and cholesterol deposition [72]. ER stress was found to be necessary to generate the M2 phenotype through JNK activation and increased PPAR $\gamma$ expression. This process was coupled with induced expression of the macrophage scavenger receptors CD36 and steroid receptor RNA activator 1 (SRA1) and increased foam cell formation. On the other hand, suppression of ER stress shifted differentiated M2 macrophages toward an M1 phenotype and subsequently suppressed foam cell formation by increasing HDL- and apoA-1-induced cholesterol efflux [72]. Moreover, M2-polarized macrophages were more sensitive to the lipotoxic effects of oxLDL than non-polarized macrophages or non-differentiated monocytic cells [73]. These studies raise the possibility that suppression of ER stress, by promoting M2 to $\mathrm{M} 1$ conversion, may reduce foam cell formation and macrophage apoptosis and thus preventing plaque progression. However, M1 macrophages are generally considered as proinflammatory cells and M2 macrophages as anti-inflammatory cells. These in vitro findings raise the interesting question of how the putative phenotypic switch between M1 and M2 macrophages is regulated in atherosclerosis and what the net effect is of these two opposing functions in disease progression.

\section{ER stress as therapeutic target}

Given the role of ER stress and UPR in the progression of atherosclerosis, therapeutic strategies to combat prolonged ER stress may have beneficial effects on preventing and alleviating the disease. Currently, agents that non-specifically relieve ER stress, such as chemical chaperones, and agents selectively targeting distinct UPR signaling molecules are under investigation in pre-clinical models. Although the mechanisms of these agents are not completely known, and not all of them have been tested in atherosclerosis-relevant models, they represent an important initial step towards targeting ER stress to treat atherosclerosis.

\section{Chemical chaperones}

So-called "chemical chaperones" have emerged as a potential therapeutic approach in multiple ER stress-related diseases. Chemical chaperones are small molecules that nonselectively stabilize mutant proteins by facilitating their proper binding. However, the majority of chemical chaperones require high concentrations to effectively prevent the misfolding of mutant proteins. These reagents at high concentration can cause non-specific 
effects and toxicity, which often makes them unsuitable for in vivo applications, especially chronic indications [74]. Currently, two chemical chaperones, namely 4-phenylbutyric acid (PBA) and tauroursodeoxycholic acid (TUDCA), are approved by the US Food and Drug Administration for use in humans $[74,75]$. Both have been recently tested in mouse models of atherosclerosis.

Western diet-fed $\mathrm{Apoe}^{-/-}$mice receiving PBA exhibited a dose-dependent reduction in atherosclerosis. Several UPR markers, including phosphorylated eIF2a, phosphorylated PERK, and ATF3, were suppressed in the lesions of mice treated with PBA, suggesting that restoring ER function can protect against the deleterious effects of toxic lipids in promoting atherosclerotic lesions. In cultured macrophages, PBA was shown to relieve saturated fatty acid-induced lipotoxicity [23], and it has also been found to alleviate glucolipotoxicity or tunicamycin-induced oxidative stress, ER stress, and apoptosis in THP-1 monocytes [76]. The effect of TUDCA treatment was tested in Western diet-fed AMP-activated protein kinase alpha 2 (Ampka2) and Ldlr double knockout mice [77]. Deletion of Ampka2 in Ldlr knockout mice enhances the progression of atherosclerotic lesions, and this effect is associated with elevated levels of ER stress markers, such as ATF6, KDEL, and XBP-1. Long term administration of TUDCA inhibited the activation of UPR markers in the lesions of these mice and suppressed the progression of atherosclerosis. Although chemical chaperones show a promising effect on relieving atherosclerosis, a definite link between their anti-atherogenic effects and ER stress-relieving function has not been established. For example, PBA has other biological actions, notably inhibition of histone deacetylases. Future studies determining whether the beneficial effects of these drugs are abrogated in genetically modified animal models with deficiency of UPR signaling molecules will be needed to test whether their anti-atherogenic effects are dependent on suppression of the UPR.

\section{Drugs targeting IRE1a}

IRE1a is an evolutionally conserved bifunctional enzyme possessing both kinase and endoribonuclease activities [5]. Elucidation of the crystal structure of yeast Ire1 [78] has promoted the discovery of pharmaceutical agents targeting this molecule. The activation of IRE1a requires dimerization. By using purified yeast Ire1 in a drug screen study, it was found that the flavonol quercetin can bind to a novel allosteric site at the dimer interface of Ire1's kinase extension nuclease domain and activate Ire1's RNase activity [79]. Quercetin and its related flavonols exert multiple functions in in vitro studies, including endotheliumindependent vasodilator effects, protective effect on nitric oxide and endothelial function under oxidative stress, suppression of platelet aggregation, inhibition of LDL oxidation, and reduction of adhesion molecules and other inflammatory markers [80]. Importantly, quercetin inhibits oxLDL-induced toxicity in endothelial cells by reducing intracellular oxidant accumulation, an effect which is mediated by the attenuation of JAK2-STAT3responsive death/survival signaling pathways involving multiple MAPKs [81]. Quercetin also prevents $\mathrm{H}_{2} \mathrm{O}_{2}$-induced apoptosis of RAW264.7 macrophages via its antioxidant activity and induction of heme oxygenase 1 gene expression [82]. Intriguingly, a recent study demonstrated that quercetin inhibits ER stress and UPR signaling in macrophages [83]. The oxLDL and tunicamycin-induced activation of UPR signaling, such as the phosphorylation of IRE1a, the nuclear translocation of ATF6, and the upregulation of XBP1 and CHOP, is generally suppressed by quercetin. This effect can be possibly interpreted by a dose-dependently attenuated oxLDL-induced cholesterol accumulation in quercetin-treated macrophages, which may facilitate the relief of ER stress [83]. Or it can be explained by the aforementioned antioxidant functions of quercetin. On the other hand, it is plausible that the selective activation of IRE1a RNase activity followed by XBP1 splicing plays a protective role, thus cutting the demands of further phosphorylation of IRE1a and expression of XBP1. 
Normally, the kinase activity of IRE1 through autophosphorylation is necessary for the ligand (ADP) binding and dimerization of IRE1 that favors its endoribonuclease activity [84, 85], while in certain circumstance this process can be bypassed [86]. Furthermore, the kinase activity of IRE1 mediates the TRAF2 recruitment and JNK activation, whereas the endoribonuclease activity of IRE1 is dispensable [56]. Interestingly, the binding of quercetin and IRE1a is independent of the phosphorylation of IRE1a [79], which may thus separate the deleterious function of its kinase activity and the potentially beneficial function of its RNase activity. Overall, which if any of the beneficial effects of quercetin depends on its activation of IRE1a RNase needs to be further investigated.

To more specifically target IRE1a activity, a peptide derived from the kinase domain of human IRE1a has been recently investigated [87]. This peptide promoted IRE1a oligomerization and enhanced its Xbpl mRNA cleavage activity in vitro in cultured human hepatocellular carcinoma cells and in Caenorhabditis elegans. The peptide attenuated both ER stress-induced JNK activation and RIDD. Cells transfected with this peptide displayed increased survival and reduced apoptosis upon tunicamycin-induced ER stress. Although the peptide has not been tested in atherosclerosis-relevant studies, the approach of targeted and selective activation of the catalytic properties of IRE1a may hold promise in this disease.

\section{Drugs targeting elF2 $\alpha$}

The phosphorylation of eIF $2 a$ suppresses global protein translation, which is thought to be a protective mechanism against excess protein client load in ER. The selective pharmacological targeting eIF2a dephosphorylation events may be useful in diseases involving client load-induced ER stress. In a screen for small molecules that protect cells from ER stress, salubrinal, a selective inhibitor of the cellular complexes that dephosphorylate eIF2a was identified [88]. Salubrinal treatment protected the rat pheochromocytoma cell line PC12 from tunicamycin-induced apoptosis in a dose-dependent manner, but did not exert inhibitory effects on non-ER stress-induced apoptosis.

Guanabenz, a a2-adrenergic receptor agonist in the treatment of hypertension, has been recently found to selectively inhibit the dephosphorylation of eIF2a by regulating growth arrest and GADD34 [89]. GADD34, which is induced by CHOP, forms a complex with the catalytic subunit of protein phospholipase 1 (PP1c), resulting in dephosphorylation of eIF2a and resumption of protein synthesis [90]. Guanabenz binds to a regulatory subunit of GADD34 and inhibits the activity of PP1c, and studies using ER-stressed cells showed that it can lower protein production rates to levels manageable by available chaperones, thus protecting cells from the lethal accumulation of misfolded proteins. Other a2-adrenergic receptor agonists tested in the same study did not affect dephosphorylation of eIF2a, indicating the effect of guanabenz on UPR is independent of its effect on the a2-adrenergic receptor.

\section{Considerations in designing drugs targeting UPR}

Several important concerns should be kept in mind when designing drugs targeting UPR signaling. Most importantly, UPR signaling is a physiological protective mechanism that protects the ER from transient disturbances. Thus, overly robust inhibition of certain UPR pathways to combat disease processes may have adverse effects on ER homeostasis in normal cells. Along these lines, different branches of the UPR may play distinct functions in any given disease process. To the extent that some branches may be protective while others are pathogenic, selective UPR branch targeting may offer promise in the therapeutic arena. Moreover, even in the same disease like atherosclerosis, different cell types, such as macrophages, ECs, and SMCs, may have distinct responses to ER stress, some of which may be protective and others detrimental. Thus, careful consideration of adverse effects 
needs to be included in UPR targeting strategies, some of which may be addressed by selective UPR branch targeting, selective cell targeting, and the identification of molecules, such as CHOP, that appear to have a higher "pathophysiologic/physiologic" index than other molecules, notably XBP1.

\section{Acknowledgments}

A.X.Z. is supported by the Swedish Research Council. I.T. is supported by NIH grants. The authors gratefully acknowledge the members of the Tabas laboratory who contributed to the studies described herein. We also thank Dr. Christopher M. Scull for his helpful discussions and valuable comments.

\section{Abbreviations}

$\begin{array}{ll}\text { Ampka2 } & \text { AMP-activated protein kinase alpha 2 } \\ \text { AP1 } & \text { Activator protein 1 } \\ \text { apoB } & \text { Apolipoprotein-B } \\ \text { ATF6 } & \text { Activating transcription factor 6 } \\ \text { BFA } & \text { Brefeldin A } \\ \text { CAMKII } & \text { Calcium/calmodulin-dependent protein kinase II } \\ \text { CASP2 } & \text { Caspase-2 } \\ \text { CHOP } & \text { CCAAT/enhancer binding protein homologous protein } \\ \text { CXCL3 } & \text { Chemokine CXC motif ligand 3 } \\ \text { DCA } & \text { Directional coronary atherectomy } \\ \text { ECs } & \text { Endothelial cells } \\ \text { eIF2a } & \text { Eukaryotic initiation factor 2a } \\ \text { ER } & \text { Endoplasmic reticulum } \\ \text { ERAD } & \text { ER-associated degradation } \\ \text { ERK } & \text { Extracellular signal-regulated kinase } \\ \text { ERO1a } & \text { ER oxidase 1a } \\ \text { FC } & \text { Free cholesterol } \\ \text { GRP78 } & \text { Glucose-regulated protein 78 } \\ \text { HHcy } & \text { Hyperhomocysteinemia } \\ \text { HSP47 } & \text { Heat shock protein } 47 \\ \text { HUVEC } & \text { Human umbilical vein endothelial cell } \\ \text { IKK } & \text { I } \text { B kinase } \\ \text { IL-6 } & \text { Interleukin-6 } \\ \text { IRE1a } & \text { Inositol-requiring protein 1 a } \\ \text { JNK } & \text { c-Jun-N-terminal kinase } \\ \text { LXR } & \text { Liver X receptor } \\ \text { MAPK } & \text { Mitogen-activated protein kinases } \\ \text { M-CSF } & \text { Macrophage colony-stimulating factor } \\ & \end{array}$




$\begin{array}{ll}\text { NLRP3 } & \text { Nucleotide oligomerization domain receptor protein } 3 \\ \text { OXLDL } & \text { Oxidized low-density lipoprotein } \\ \text { PBA } & \text { 4-Phenylbutyric acid } \\ \text { PERK } & \text { Protein kinase RNA-like ER kinase } \\ \text { PP1c } & \text { Protein phospholipase 1, catalytic subunit } \\ \text { PRRs } & \text { Pattern recognition receptors } \\ \text { RIDD } & \text { IRE1-dependent decay } \\ \text { SAP } & \text { Stable angina pectoris } \\ \text { SMCs } & \text { Smooth muscle cells } \\ \text { SRA1 } & \text { Steroid receptor RNA activator 1 } \\ \text { STAT1 } & \text { Signal transducer and activator of transcription-1 } \\ \text { SXBP1 } & \text { Spliced XBP1 protein } \\ \text { TDAG51 } & \text { T cell death associated gene 51 } \\ \text { tHcy } & \text { Total serum homocysteine } \\ \text { TLRs } & \text { Toll-like receptors } \\ \text { TRAF2 } & \text { TNFR-associated factor 2 } \\ \text { TNFa } & \text { Tumor necrosis factor-a } \\ \text { TUDCA } & \text { Tauroursodeoxycholic acid } \\ \text { TXNIP } & \text { Thioredoxin-interacting protein } \\ \text { UPR } & \text { Unfolded protein response } \\ \text { XBP1 } & \text { Xnstable angina pectoris } \\ \text { UAP } & \end{array}$

\section{References}

1. Murray CJ, Lopez AD. Global mortality, disability, and the contribution of risk factors: Global Burden of Disease Study. Lancet. 1997; 349(9063):1436-1442. [PubMed: 9164317]

2. Rader DJ, Daugherty A. Translating molecular discoveries into new therapies for atherosclerosis. Nature. 2008; 451(7181):904-913. [PubMed: 18288179]

3. Tabas I. The role of endoplasmic reticulum stress in the progression of atherosclerosis. Circ Res. 2010; 107(7):839-850. [PubMed: 20884885]

4. Minamino T, Komuro I, Kitakaze M. Endoplasmic reticulum stress as a therapeutic target in cardiovascular disease. Circ Res. 2010; 107(9):1071-1082. [PubMed: 21030724]

5. Ron D, Walter P. Signal integration in the endoplasmic reticulum unfolded protein response. Nat Rev Mol Cell Biol. 2007; 8(7):519-529. [PubMed: 17565364]

6. Szegezdi E, et al. Mediators of endoplasmic reticulum stress-induced apoptosis. EMBO Rep. 2006; 7(9):880-885. [PubMed: 16953201]

7. Hetz C. The unfolded protein response: controlling cell fate decisions under ER stress and beyond. Nat Rev Mol Cell Biol. 2012; 13(2):89-102. [PubMed: 22251901]

8. Williams KJ, Tabas I. The response-to-retention hypothesis of atherogenesis reinforced. Curr Opin Lipidol. 1998; 9(5):471-474. [PubMed: 9812202] 
9. Tabas I, Williams KJ, Boren J. Subendothelial lipoprotein retention as the initiating process in atherosclerosis: update and therapeutic implications. Circulation. 2007; 116(16):1832-1844. [PubMed: 17938300]

10. Doran AC, Meller N, McNamara CA. Role of smooth muscle cells in the initiation and early progression of atherosclerosis. Arterioscler Thromb Vasc Biol. 2008; 28(5):812-819. [PubMed: 18276911]

11. Hansson GK. Inflammation, atherosclerosis, and coronary artery disease. N Engl J Med. 2005; 352(16):1685-1695. [PubMed: 15843671]

12. Fuster V. Lewis A. Conner Memorial Lecture. Mechanisms leading to myocardial infarction: insights from studies of vascular biology. Circulation. 1994; 90(4):2126-2146. [PubMed: 7718033]

13. Tabas I. Consequences and therapeutic implications of macrophage apoptosis in atherosclerosis: the importance of lesion stage and phagocytic efficiency. Arterioscler Thromb Vasc Biol. 2005; 25(11):2255-2264. [PubMed: 16141399]

14. Bennett MR. Apoptosis of vascular smooth muscle cells in vascular remodelling and atherosclerotic plaque rupture. Cardiovasc Res. 1999; 41(2):361-368. [PubMed: 10341835]

15. Rutkowski DT, Hegde RS. Regulation of basal cellular physiology by the homeostatic unfolded protein response. J Cell Biol. 2010; 189(5):783-794. [PubMed: 20513765]

16. Myoishi M, et al. Increased endoplasmic reticulum stress in atherosclerotic plaques associated with acute coronary syndrome. Circulation. 2007; 116(11):1226-1233. [PubMed: 17709641]

17. Zhou J, et al. Activation of the unfolded protein response occurs at all stages of atherosclerotic lesion development in apolipoprotein E-deficient mice. Circulation. 2005; 111(14):1814-1821. [PubMed: 15809369]

18. Thorp E, et al. Reduced apoptosis and plaque necrosis in advanced atherosclerotic lesions of Apoe -/- and Ldlr-/- mice lacking CHOP. Cell Metab. 2009; 9(5):474-481. [PubMed: 19416717]

19. Hossain GS, et al. TDAG51 is induced by homocysteine, promotes detachment-mediated programmed cell death, and contributes to the development of atherosclerosis in hyperhomocysteinemia. J Biol Chem. 2003; 278(32):30317-30327. [PubMed: 12738777]

20. Maxfield FR, Tabas I. Role of cholesterol and lipid organization in disease. Nature. 2005; 438(7068):612-621. [PubMed: 16319881]

21. Li Y, et al. Enrichment of endoplasmic reticulum with cholesterol inhibits sarcoplasmicendoplasmic reticulum calcium ATPase- $2 b$ activity in parallel with increased order of membrane lipids: implications for depletion of endoplasmic reticulum calcium stores and apoptosis in cholesterol-loaded macrophages. J Biol Chem. 2004; 279(35):37030-37039. [PubMed: 15215242]

22. Fu Y, Luo N, Lopes-Virella MF. Oxidized LDL induces the expression of ALBP/aP2 mRNA and protein in human THP-1 macrophages. J Lipid Res. 2000; 41(12):2017-2023. [PubMed: 11108735]

23. Erbay E, et al. Reducing endoplasmic reticulum stress through a macrophage lipid chaperone alleviates atherosclerosis. Nat Med. 2009; 15(12):1383-1391. [PubMed: 19966778]

24. Makowski L, et al. Lack of macrophage fatty-acid-binding protein aP2 protects mice deficient in apolipoprotein E against atherosclerosis. Nat Med. 2001; 7(6):699-705. [PubMed: 11385507]

25. Civelek M, et al. Coronary artery endothelial transcriptome in vivo: identification of endoplasmic reticulum stress and enhanced reactive oxygen species by gene connectivity network analysis. Circ Cardiovasc Genet. 2011; 4(3):243-252. [PubMed: 21493819]

26. Civelek M, et al. Chronic endoplasmic reticulum stress activates unfolded protein response in arterial endothelium in regions of susceptibility to atherosclerosis. Circ Res. 2009; 105(5):453461. [PubMed: 19661457]

27. Zeng L, et al. Sustained activation of XBP1 splicing leads to endothelial apoptosis and atherosclerosis development in response to disturbed flow. Proc Natl Acad Sci U S A. 2009; 106(20):8326-8331. [PubMed: 19416856]

28. Cesari M, et al. Is homocysteine important as risk factor for coronary heart disease? Nutr Metab Cardiovasc Dis. 2005; 15(2):140-147. [PubMed: 15871863]

29. Eikelboom JW, et al. Homocyst(e)ine and cardiovascular disease: a critical review of the epidemiologic evidence. Ann Intern Med. 1999; 131(5):363-375. [PubMed: 10475890] 
30. Zhou J, Austin RC. Contributions of hyperhomocysteinemia to atherosclerosis: causal relationship and potential mechanisms. Biofactors. 2009; 35(2):120-129. [PubMed: 19449439]

31. Zhou J, et al. Association of multiple cellular stress pathways with accelerated atherosclerosis in hyperhomocysteinemic apolipoprotein E-deficient mice. Circulation. 2004; 110(2):207-213. [PubMed: 15210586]

32. Zhou J, et al. Hyperhomocysteinemia induced by methionine supplementation does not independently cause atherosclerosis in C57BL/6J mice. FASEB J. 2008; 22(7):2569-2578. [PubMed: 18364397]

33. Zulli A, et al. High dietary taurine reduces apoptosis and atherosclerosis in the left main coronary artery: association with reduced CCAAT/enhancer binding protein homologous protein and total plasma homocysteine but not lipidemia. Hypertension. 2009; 53(6):1017-1022. [PubMed: 19398656]

34. Tabas I, Ron D. Integrating the mechanisms of apoptosis induced by endoplasmic reticulum stress. Nat Cell Biol. 2011; 13(3):184-190. [PubMed: 21364565]

35. Dickhout JG, et al. Induction of the unfolded protein response after monocyte to macrophage differentiation augments cell survival in early atherosclerotic lesions. FASEB J. 2010; 25(2):576589. [PubMed: 20966213]

36. Tsukano $\mathrm{H}$, et al. The endoplasmic reticulum stress-C/EBP homologous protein pathway-mediated apoptosis in macrophages contributes to the instability of atherosclerotic plaques. Arterioscler Thromb Vasc Biol. 2010; 30(10):1925-1932. [PubMed: 20651282]

37. Gao J, et al. Involvement of endoplasmic stress protein C/EBP homologous protein in arteriosclerosis acceleration with augmented biological stress responses. Circulation. 2011; 124(7): 830-9. [PubMed: 21810656]

38. Feng B, et al. The endoplasmic reticulum is the site of cholesterol-induced cytotoxicity in macrophages. Nat Cell Biol. 2003; 5(9):781-92. [PubMed: 12907943]

39. Sun Y, et al. Free cholesterol accumulation in macrophage membranes activates Toll-like receptors and p38 mitogen-activated protein kinase and induces cathepsin K. Circ Res. 2009; 104(4):455465. [PubMed: 19122179]

40. Li G, et al. Role of ERO1-alpha-mediated stimulation of inositol 1,4,5-triphosphate receptor activity in endoplasmic reticulum stress-induced apoptosis. J Cell Biol. 2009; 186(6):783-792. [PubMed: 19752026]

41. Timmins JM, et al. Calcium/calmodulin-dependent protein kinase II links ER stress with Fas and mitochondrial apoptosis pathways. J Clin Invest. 2009; 119(10):2925-2941. [PubMed: 19741297]

42. Seimon TA, et al. Atherogenic lipids and lipoproteins trigger CD36-TLR2-dependent apoptosis in macrophages undergoing endoplasmic reticulum stress. Cell Metab. 2010; 12(5):467-482. [PubMed: 21035758]

43. McCullough KD, et al. Gadd153 sensitizes cells to endoplasmic reticulum stress by downregulating Bcl2 and perturbing the cellular redox state. Mol Cell Biol. 2001; 21(4):1249-1259. [PubMed: 11158311]

44. Halterman MW, et al. Loss of c/EBP-beta activity promotes the adaptive to apoptotic switch in hypoxic cortical neurons. Mol Cell Neurosci. 2008; 38(2):125-137. [PubMed: 18439838]

45. Chiribau CB, et al. Molecular symbiosis of CHOP and C/EBP beta isoform LIP contributes to endoplasmic reticulum stress-induced apoptosis. Mol Cell Biol. 2010; 30(14):3722-3731. [PubMed: 20479126]

46. Puthalakath $\mathrm{H}$, et al. ER stress triggers apoptosis by activating BH3-only protein Bim. Cell. 2007; 129(7):1337-1349. [PubMed: 17604722]

47. Ghosh AP, et al. CHOP potentially co-operates with FOXO3a in neuronal cells to regulate PUMA and BIM expression in response to ER stress. PLoS One. 2012; 7(6):e39586. [PubMed: 22761832]

48. Marciniak SJ, et al. CHOP induces death by promoting protein synthesis and oxidation in the stressed endoplasmic reticulum. Genes Dev. 2004; 18(24):3066-3077. [PubMed: 15601821]

49. Seimon TA, et al. Macrophage deficiency of p38alpha MAPK promotes apoptosis and plaque necrosis in advanced atherosclerotic lesions in mice. J Clin Invest. 2009; 119(4):886-898. [PubMed: 19287091] 
50. Seimon TA, et al. Combinatorial pattern recognition receptor signaling alters the balance of life and death in macrophages. Proc Natl Acad Sci U S A. 2006; 103(52):19794-19799. [PubMed: 17167049]

51. Zhang C, et al. Homocysteine induces programmed cell death in human vascular endothelial cells through activation of the unfolded protein response. J Biol Chem. 2001; 276(38):35867-35874. [PubMed: 11447214]

52. Dong Y, et al. Activation of AMP-activated protein kinase inhibits oxidized LDL-triggered endoplasmic reticulum stress in vivo. Diabetes. 2010; 59(6):1386-1396. [PubMed: 20299472]

53. Pedruzzi E, et al. NAD(P)H oxidase Nox-4 mediates 7-ketocholesterol-induced endoplasmic reticulum stress and apoptosis in human aortic smooth muscle cells. Mol Cell Biol. 2004; 24(24): 10703-10717. [PubMed: 15572675]

54. Kedi X, et al. Free cholesterol overloading induced smooth muscle cells death and activated both ER- and mitochondrial-dependent death pathway. Atherosclerosis. 2009; 207(1):123-130. [PubMed: 19467656]

55. Han D, et al. IRE1alpha kinase activation modes control alternate endoribonuclease outputs to determine divergent cell fates. Cell. 2009; 138(3):562-575. [PubMed: 19665977]

56. Urano F, et al. Coupling of stress in the ER to activation of JNK protein kinases by transmembrane protein kinase IRE1. Science. 2000; 287(5453):664-666. [PubMed: 10650002]

57. Lei K, Davis RJ. JNK phosphorylation of Bim-related members of the Bcl 2 family induces Baxdependent apoptosis. Proc Natl Acad Sci U S A. 2003; 100(5):2432-2437. [PubMed: 12591950]

58. Yamamoto K, Ichijo H, Korsmeyer SJ. BCL-2 is phosphorylated and inactivated by an ASK1/Jun N-terminal protein kinase pathway normally activated at G(2)/M. Mol Cell Biol. 1999; 19(12): 8469-8478. [PubMed: 10567572]

59. Upton JP, et al. IRE1alpha cleaves select microRNAs during ER stress to derepress translation of proapoptotic caspase-2. Science. 2012 doi:10.3410/f.717959717.793462943.

60. Bonzon C, et al. Caspase-2-induced apoptosis requires bid cleavage: a physiological role for bid in heat shock-induced death. Mol Biol Cell. 2006; 17(5):2150-2157. [PubMed: 16495337]

61. Lerner AG, et al. IRE1alpha induces thioredoxin-interacting protein to activate the NLRP3 inflammasome and promote programmed cell death under irremediable ER stress. Cell Metab. 2012; 16(2):250-264. [PubMed: 22883233]

62. Zhang K, Kaufman RJ. From endoplasmic-reticulum stress to the inflammatory response. Nature. 2008; 454(7203):455-462. [PubMed: 18650916]

63. Hotamisligil GS. Endoplasmic reticulum stress and the inflammatory basis of metabolic disease. Cell. 2010; 140(6):900-917. [PubMed: 20303879]

64. Li Y, et al. Free cholesterol-loaded macrophages are an abundant source of tumor necrosis factoralpha and interleukin-6: model of NF-kappaB- and map kinase-dependent inflammation in advanced atherosclerosis. J Biol Chem. 2005; 280(23):21763-21772. [PubMed: 15826936]

65. Menu P, et al. ER stress activates the NLRP3 inflammasome via an UPR-independent pathway. Cell Death Dis. 2012; 3:e261. [PubMed: 22278288]

66. Kim JB, et al. Paraoxonase-2 modulates stress response of endothelial cells to oxidized phospholipids and a bacterial quorum-sensing molecule. Arterioscler Thromb Vasc Biol. 2011; 31(11):2624-2633. [PubMed: 21836061]

67. Gargalovic PS, et al. Identification of inflammatory gene modules based on variations of human endothelial cell responses to oxidized lipids. Proc Natl Acad Sci U S A. 2006; 103(34):1274112746. [PubMed: 16912112]

68. Gargalovic PS, et al. The unfolded protein response is an important regulator of inflammatory genes in endothelial cells. Arterioscler Thromb Vasc Biol. 2006; 26(11):2490-2496. [PubMed: 16931790]

69. Porcheray F, et al. Macrophage activation switching: an asset for the resolution of inflammation. Clin Exp Immunol. 2005; 142(3):481-489. [PubMed: 16297160]

70. Fleming BD, Mosser DM. Regulatory macrophages: setting the threshold for therapy. Eur J Immunol. 2011; 41(9):2498-2502. [PubMed: 21952805]

71. Khallou-Laschet J, et al. Macrophage plasticity in experimental atherosclerosis. PLoS One. 2010; 5(1):e8852. [PubMed: 20111605] 
72. Oh J, et al. Endoplasmic reticulum stress controls M2 macrophage differentiation and foam cell formation. J Biol Chem. 2012; 287(15):11629-116241. [PubMed: 22356914]

73. Isa SA, et al. M2 macrophages exhibit higher sensitivity to oxLDL-induced lipotoxicity than other monocyte/macrophage subtypes. Lipids Health Dis. 2011; 10:229. [PubMed: 22146099]

74. Engin F, Hotamisligil GS. Restoring endoplasmic reticulum function by chemical chaperones: an emerging therapeutic approach for metabolic diseases. Diabetes Obes Metab. 2010; 12(Suppl 2): 108-115. [PubMed: 21029307]

75. Ozcan U, et al. Chemical chaperones reduce ER stress and restore glucose homeostasis in a mouse model of type 2 diabetes. Science. 2006; 313(5790):1137-1140. [PubMed: 16931765]

76. Lenin R, et al. Amelioration of glucolipotoxicity-induced endoplasmic reticulum stress by a "chemical chaperone" in human THP-1 monocytes. Exp Diabetes Res. 2012; 2012:356487. [PubMed: 22550476]

77. Dong Y, et al. Reduction of AMP-activated protein kinase alpha2 increases endoplasmic reticulum stress and atherosclerosis in vivo. Circulation. 2010; 121(6):792-803. [PubMed: 20124121]

78. Credle JJ, et al. On the mechanism of sensing unfolded protein in the endoplasmic reticulum. Proc Natl Acad Sci U S A. 2005; 102(52):18773-18734. [PubMed: 16365312]

79. Wiseman RL, et al. Flavonol activation defines an unanticipated ligand-binding site in the kinaseRNase domain of IRE1. Mol Cell. 2010; 38(2):291-304. [PubMed: 20417606]

80. Perez-Vizcaino F, Duarte J. Flavonols and cardiovascular disease. Mol Aspects Med. 2010; 31(6): 478-494. [PubMed: 20837053]

81. Choi JS, et al. Blockade of oxidized LDL-triggered endothelial apoptosis by quercetin and rutin through differential signaling pathways involving JAK2. J Agric Food Chem. 2009; 57(5):2079_ 2086. [PubMed: 19196000]

82. Chow JM, et al. Quercetin, but not rutin and quercitrin, prevention of $\mathrm{H} 2 \mathrm{O} 2$-induced apoptosis via anti-oxidant activity and heme oxygenase 1 gene expression in macrophages. Biochem Pharmacol. 2005; 69(12):1839-51. [PubMed: 15876423]

83. Yao S, et al. Quercetin protects macrophages from oxidized low-density lipoprotein-induced apoptosis by inhibiting the endoplasmic reticulum stress-C/EBP homologous protein pathway. Exp Biol Med (Maywood). 2012; 237(7):822-831. [PubMed: 22829699]

84. Cox JS, Shamu CE, Walter P. Transcriptional induction of genes encoding endoplasmic reticulum resident proteins requires a transmembrane protein kinase. Cell. 1993; 73(6):1197-11206. [PubMed: 8513503]

85. Mori K, et al. A transmembrane protein with a cde2+/CDC28-related kinase activity is required for signaling from the ER to the nucleus. Cell. 1993; 74(4):743-756. [PubMed: 8358794]

86. Papa FR, et al. Bypassing a kinase activity with an ATP-competitive drug. Science. 2003; 302(5650):1533-1537. [PubMed: 14564015]

87. Bouchecareilh M, et al. Peptides derived from the bifunctional kinase/RNase enzyme IRE1alpha modulate IRE1alpha activity and protect cells from endoplasmic reticulum stress. FASEB J. 2011; 25(9):3115-3129. [PubMed: 21680894]

88. Boyce M, et al. A selective inhibitor of eIF2alpha dephosphorylation protects cells from ER stress. Science. 2005; 307(5711):935-939. [PubMed: 15705855]

89. Tsaytler $\mathrm{P}$, et al. Selective inhibition of a regulatory subunit of protein phosphatase 1 restores proteostasis. Science. 2011; 332(6025):91-94. [PubMed: 21385720]

90. Novoa I, et al. Feedback inhibition of the unfolded protein response by GADD34-mediated dephosphorylation of eIF2alpha. J Cell Biol. 2001; 153(5):1011-1012. [PubMed: 11381086] 


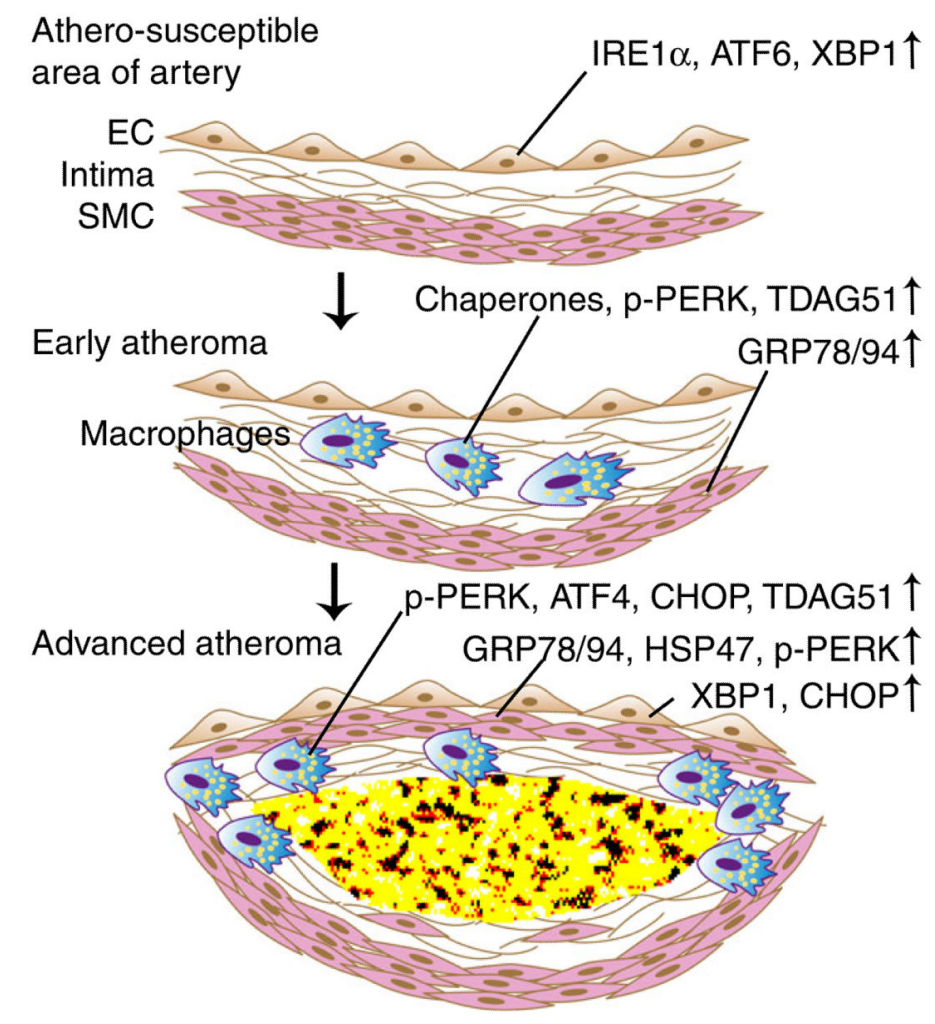

Fig. 1.

Sequential activation of UPR signaling pathways in atherosclerosis. Endothelial cells (ECs) in athero-susceptible regions of pre-lesional arteries are primed for expression of ER chaperones with induced expression of ATF6, IRE1a, and XBP1. ER chaperones are expressed in both early and advanced atheroma, most likely as a compensatory response to restore ER homeostasis. The PERK pathway is activated in macrophages at all stages of atherosclerosis and in ECs and SMCs in advanced lesions. The early activation of the PERK pathway might be protective through suppression of protein synthesis, which reduces ER protein client load. However, sustained and enhanced PERK activation induces CHOP in advanced lesions, leading to apoptosis. Similarly, the early expression of XBP1 in ECs promotes ER chaperone expression, while it likely becomes detrimental in advanced lesions. Despite a paucity of evidence in vivo, XBP1 splicing in advanced lesions indicates IRE1a activation, which may promote apoptosis through other nuclease actions. Please see text for specific details 